\title{
Kontrolle der oralen Antikoagulantientherapie I: Standardisierung der Hepato Quick-Citratplasma- Methode nach dem WHO-Modell in einer multizentrischen Studie
}

\author{
E. Spanuth ${ }^{7}$, H. Beeser ${ }^{1}$, F. Keller ${ }^{2}$, H. Köstering ${ }^{3}$, W. Müller-Beißenhirtz ${ }^{4}$, G. Pindur ${ }^{5}$, S. Popov-Cenic ${ }^{6}$, E. Zimmer $^{7}$ \\ Zentrale Einrichtung Transfusionsmedizin der Universität Freiburg \\ 2 Zentrallabor der Medizinischen Universitätsklinik Würzburg \\ 3 Medizinische Universitätsklinik Göttingen \\ 4 Klinisch-Chemische Abteilung, Bürgerhospital Stuttgart \\ 5 Abteilung für klinische Hämostaseologie und Transfusionsmedizin der Universitätskliniken Homburg \\ 6 Institut für Experimentelle Hämatologie und Bluttransfusionswesen der Universität Bonn \\ 7 Boehringer Mannheim GmbH, Abt. Blutgerinnung
}

Zusammenfassung:

Hepato Quick, ein nach Owren modifiziertes Thromboplastinreagenz, das bariumsulfatadsorbiertes Rinderplasma enthält, wurde gegen das internationale Referenzthromboplastin OBT/79 in einer multizentrischen Studie nach dem von der WHO empfohlenen Verfahren kalibriert. Die einzige Abweichung von diesem Verfahren bestand darin, daß nicht die Kipp-, sondern die Häkchenmethode unter Verwendung des Koagulometers nach Schnitger und Gross angewandt wurde, da dieses Verfahren eine bessere Reproduzierbarkeit und Präzision gewährleistet. Die Kipp-Methode wurde lediglich in einem Labor angewandt. Jeder der fünf Teilnehmer hat die Kalibrierung von zwei Reagenzchargen Hepato Quick durchgeführt. Eine Charge war bei allen Teilnehmern identisch (Hausstandard), während die zweite Charge in jedem Labor verschieden war. Für die Hausstandard-Charge wurde ein International Sensitivity Index (ISI) von 0,95 mit einem 2s-Bereich von $\pm 0,08$ ermittelt. Für die fünf verschiedenen Reagenzchargen wurde ebenfalls ein mittlerer ISI von 0,95 mit einem $2 s$-Bereich von $\pm 0,054$ ermittelt. Die Standardisierung gegen das WHO-Referenzthromboplastin $O B T / 79$ sowie gegen den etablierten Hausstandard liefert gleiche ISI-Wèrte innerhalb der meßtechnischen Schwankungsbreite. Die Ergebnisse zeigen, daß das WHO-Modell zur Standardisierung der Thromboplastinzeit voll auf Hepato Quick angewandt werden kann.

Schlüsse/wörter:

Thromboplastinzeit - Orale Antikoagulantientherapie - Hepato Quick - Standardisierung - International Sensitivity Index (ISI) - International Normalized Ratio (INR)

Summary:

Hepato Quick, a modified thromboplastin containing adsorbed bovine plasma in addition to the thromboplastin, was calibrated against the international reference thromboplastin OBT/79 in a multicentre study based on the model proposed by the WHO. The only deviation from the WHO procedure was that measurements were generally made not with the tilt method but with the hook method using the Schnitger and Gross coagulometer as this procedure provides better precision and reproducibility of the results. The tilt method was only used by one laboratory. Each of the 5 participating laboratories has calibrated two batches of Hepato Quick. One of the batches was the same for all the laboratories (house standard) whereas the second batch was different for each laboratory. For the house standard an international sensitivity index (ISI) of 0.95 and an inter-laboratory scatter range (2s) of 0.08 was obtained. In agreement with this, values of 0.95 and 0.054 respectively were found for the 5 different batches of Hepato Quick. Upon calibration of these 5 hatches against the international reference thromboplastin OBT/79 and on the other hand against the established house standard, it can be seen that identical values for ISI are obtained on both ways. So it is evident that the WHO model is well suited for standardisation of Hepato Quick.

Keywords:

Prothrombin time - oral anticoagulant therapy - Hepato Quick - Standardisation - International Sensitivity Index (ISI) - International Normalized Ratio (INR) 


\section{Einleitung}

Die orale Antikoagulation mit Cumarinderivaten ist eine etablierte und wirksame Therapie zur Thromboseprophylaxe. Unterschiedliche Auffassungen bestehen lediglich zu einzelnen Indikationen und der Therapiedauer. Als absolute Indikationen gelten in der Literatur übereinstimmend Folgezustände nach tiefen Bein- und Beckenvenenthrombosen (ca. 6 Monate) sowie nach Lungenembolie (ca. 12 Monate) $(8,20)$. Eine Dauerantikoagulation ist notwendig bei Vorhofflimmern mit arteriellen Embolien, bei Kardiomyopathien mit Hyperkinesie der Ventrikelmuskulatur, bei Herzklappenersatz mit Ausnahme der Bioprothesen sowie beim hereditären Antithrombin III-, Protein-C- und Protein-S-Mangel. Kontrovers diskutiert wird dagegen die Behandlung mit oralen Antikoagulantien nach Herzinfarkt. Inzwischen liegen jedoch verschiedene Studien vor, in denen die Effektivität der oralen Antikoagulation eindeutig belegt werden konnte. Hervorzuheben sind hier die Studien des Holländischen Thrombosedienstes $(1,14)$. In der sog. Sixty Plus-Studie (1) konnte gezeigt werden, daß auch bei Patienten über 60 Jahre die Mortalität und Reinfarkthäufigkeit durch eine Dauerantikoagulation signifikant gesenkt werden kann.

Der Erfolg der oralen Antikoagulantientherapie ist primär abhängig von der Intensität der Antikoagulation und der optimalen Einstellung des Patienten $(10,15,17)$. Dies bedeutet, daß Kontrolle und Steuerung der Therapie über die Bestimmung der Thromboplastinzeit nach Quick den Therapieerfolg wesentlich beeinflussen. Als Hauptprobleme bei der Thromboplastinzeitbestimmung zur Kontrolle der oralen Antikoagulantientherapie haben sich die unterschiedlichen Empfindlichkeiten und die dadurch bedingten unterschiedlichen therapeutischen Bereiche der kommerziell verfügbaren Thromboplastinreagenzien erwiesen $(4,18,19)$. Eine direkte Vergleichbarkeit der Werte ist häufig nicht gegeben. Dies kann zu Unterdosierungen führen, wenn Patienten an verschiedenen Orten mit unterschiedlichen Reagenzien kontrolliert werden. Die Folgen der mangelnden Vergleichbarkeit aufgrund der unterschiedlichen Empfindlichkeit verschiedener Thromboplastine sind

- unterschiedliche therapeutische Bereiche in Prozent,

- mögliche Verstärkung der Differenzen durch fehlerhafte Kalibrierung,

- unterschiedliche Antikoagulation.

Um diese Situation zu verbessern, wird schon seit Jahren von internationalen Gremien die Standardisierung der Thromboplastinreagenzien angestrebt. Daneben wird auf

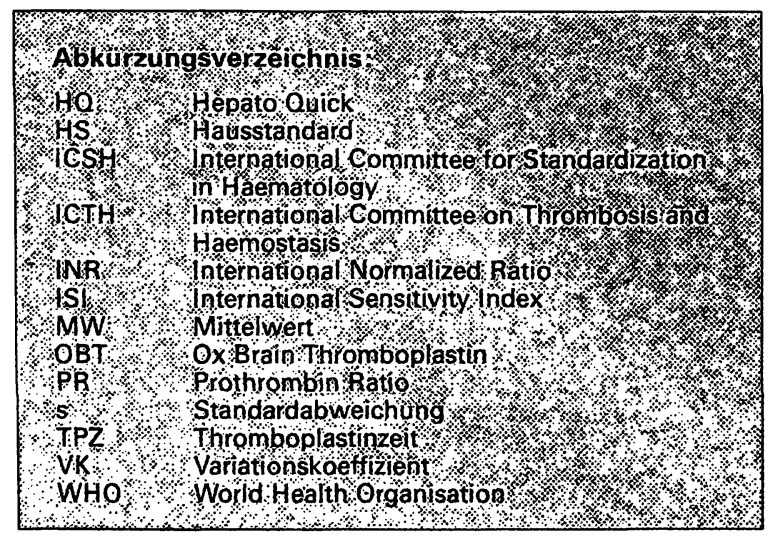

nationaler Ebene, vor allem in Deutschland (3) und Großbritannien (31), versucht, die Thromboplastinzeitbestimmung durch externe Ringversuche und standardisierte Kalibrierverfahren [chargen- und gerätespezifische Wertetabellen (23)] zu verbessern.

Vom International Committee on Thrombosis and Haemostasis (ICTH) und dem International Committee for Standardization in Haematology (ICSH) wurde 1983 ein System vorgestellt, das erstmals einen erfolgversprechenden Ansatz für eine Standardisierung der Thromboplastinreagenzien darstellt und von der WHO übernommen wurde (31). Das besondere Prinzip dieses Systems besteht darin, daß die verschiedenen Thromboplastinreagenzien an einer festgelegten Charge eines Referenzthromboplastins geeicht werden. Das Ergebnis der Thromboplastinzeitbestimmung wird dabei jedoch nicht wie in Deutschland und anderen europäischen Ländern in "Prozent der Norm”, sondern wie im angelsächsischen Raum üblich, als "Ratio" angegeben ["Ratio" (PR) $=$ Thromboplastinzeit des Patienten dividiert durch Thromboplastinzeit eines Normalplasmas]. Als Urstandard wurde das internationale Referenzthromboplastin mit der Bezeichnung 67/40 etabliert. Die „Ratio”, die man mit diesem Thromboplastin erhält, wird als ,International Normalized Ratio“ (INR) bezeichnet. Für die verschiedenen kommerziellen Thromboplastine sollen durch Vergleich mit dem Referenzthromboplastin chargenspezifische Faktoren ermittelt werden, die die Umrechnung in INR und damit eine direkte Vergleichbarkeit der mit verschiedenen Reagenzien ermittelten Werte ermöglichen. Hierzu werden an 10 Tagen von jeweils 6 stabil eingestellten Antikoagulantienpatienten (insgesamt 60) und zwei Normalprobanden (insgesamt 20) die Thromboplastinzeiten mit dem internationalen Referenzthromboplastin und der zu standardisierenden ThromboplastinReagenzcharge bestimmt und in einem log/log-System gegeneinander aufgetragen. Durch die Meßpunkte wird eine Ausgleichsgerade gelegt, deren Steigung mit "International Sensitivity Index" (ISI) bezeichnet wird. Bei bekanntem ISI ist es möglich, die mit einem beliebigen Thromboplastin ermittelte "Ratio" eines Patienten (PR) in die INR nach folgender Formel umzurechnen $(10,29)$ : INR $=$ PR $^{\text {ISI }}$

Mit diesem Verfahren können Patientenwerte, die streng genommen mit beliebigen Reagenzien ermittelt wurden, in die universalgültige INR-Skala umgerechnet werden. Es ist zu erwähnen, daß das System nur gültig ist für Patienten in der stabilen Phase einer oralen Antikoagulantientherapie.

Besondere Bedeutung kommt der Festlegung des ISI zu. Dieser Wert muß vom Reagenzienhersteller für jede Reagenzcharge entsprechend dem von der ICTH/ICSH vorgegebenen Procedere ermittelt werden. Erste Erfahrungen haben gezeigt, daß einige Einschränkungen gemacht werden müssen. So ist der ISI nicht nur von der Produktionscharge des Thromboplastins, sondern u.a. auch vom Meßprinzip bzw. von dem Gerätetyp abhängig (30).

\section{Das Hepato Quick-System}

Obwohl die Thromboplastinzeitbestimmung, insbesondere in der Klinik, neben ihrer Anwendung als globale Screeningmethode bevorzugt für die Kontrolle der oralen Antikoagulation eingesetzt wird, dürfen einige grundsätzliche Schwachpunkte nicht übersehen werden. Aufgrund des geringen Probe-Reagenz-Volumenverhältnisses (in der Regel 1:3) resultieren relativ kurze Meßzeiten, 
deren Variabilität eine sehr sorgfältige Arbeitsweise bei Probengewinnung und Testdurchführung erfordert, wenn eine ausreichende Reproduzierbarkeit der Ergebnisse gewährleistet werden soll (26). Außerdem ist die Thromboplastinzeit nicht nur von den durch orale Antikoagulantien beeinflußten Faktoren, sondern zusätzlich auch vom lagerungsinstabilen. Faktor $V$ und Fibrinogen abhängig. Dies ist bei der Kontrolle der oralen Antikoagulantientherapie nicht erwünscht.

Aus diesen Gründen wurde von P. A. Owren bereits 1951 auf der Basis der P.- und P.- Methode (21) der Thrombotest als Faktor II-, VII- und X-empfindliche Methode entwickelt. Das Reagenz besteht aus Rinderhirnthromboplastin, dem Faktor V und Fibrinogen in Form von bariumsulfatadsorbiertem Rinderoxalatplasma zugesetzt ist. Aufgrund eines durch den Fibrinogenzusatz möglichen größeren Probe-Reagenz-Volumenverhältnisses werden längere Gerinnungszeiten gemessen als bei der konventionellen Thromboplastinzeitbestimmung. Als Probenmaterial kann sowohl Vollblut als auch Citratplasma eingesetzt werden (22). Die Anwendbarkeit dieser Methode ist allerdings auf den Meßbereich unterhalb $50 \%$ der Norm beschränkt, so daß für die Abdeckung des Normalbereiches eine Modifikation unter der Bezeichnung "Normotest" eingeführt wurde (23).

Als Weiterentwicklung beider Methoden ist Hepato Quick anzusehen (6). Das Reagenz besteht aus Primatenhirnund Kaninchenhirnthromboplastin im Verhältnis 1:1 und bariumsulfatadsorbiertem Rinderoxalatplasma als Fibrinogen- und Faktor-V-Quelle. Der therapeutische Bereich wurde mit $10-20 \%$ der Norm ermittelt $(2,6,7)$. Das Reagenz ist im gesamten Meßbereich ( 0 bis $>100 \%$ der Norm) anwendbar. Sowohl im Vergleich zu „Thrombotest" mit Plasmen von oral antikoagulierten Patienten als auch im Vergleich zu „Normotest“ mit Plasmen von Patienten mit normaler Blutgerinnung ergeben sich jeweils gute Übereinstimmungen mit den Resultaten des Hepato Quick (6). Ringversuche belegen, daß sowohl im Normalbereich als auch im therapeutischen Bereich eine gute Wiederfindung und Vergleichbarkeit von Labor zu Labor erhalten wird (28). Die Bestimmung kann mit venösem Citratplasma, Kapillar-Citratblut und Kapillar-Vollblut durchgeführt werden, so daß die den Patienten belastende Venenpunktion vermieden werden kann. Durch den Zusatz von bariumsulfatadsorbiertem Rinderoxalatplasma ergeben sich längere Haltbarkeiten des Probenmaterials von bis zu 48 Std.

\section{Zielsetzung der Studie}

\subsection{Etablierung eines Hausstandards für Hepato Quick}

$\mathrm{Da}$ die Referenzthromboplastine nicht in beliebiger Menge zur Verfügung stehen, muß vom Hersteller eine Reagenziencharge ausgewählt und als sogenannter Hausstandard etabliert werden. Dieser Hausstandard wird zur Ermittlung der ISI-Werte für die Chargen aus der laufenden Produktion eingesetzt. Im Rahmen der vorliegenden mulrizentrischen Studie sollte in allen beteiligten Laboratorien für die als Hausstandard ausgewählte Hepato Quick-Charge der ISI bestimmt werden.

\subsection{Ermittlung der Chargenvarianz des ISI von Hepato Quick}

Neben der als Hausstandard vorgesehenen Reagenzcharge sollte von einer zweiten, für jeden Teilnehmer un- terschiedlichen Charge Hepato Quick der ISI ermittelt werden. Mit den Daten über 6 verschiedene Hepato Quick-Chargen aus voneinander unabhängigen Laboratorien soll eine Aussage über die Chargenvarianz des ISI von Hepato Quick gemacht werden.

\subsection{Ermittlung des Einflusses des Meßprinzips auf den ISI}

Bei der von der ICTH/ICSH durchgeführten Standardisierung der sekundären Referenzthromboplastine wurde zur Bestimmung der Thromboplastinzeiten die sogenannte Kipptechnik angewendet, die auch für die Hersteller zur Kalibrierung ihrer Reagenzien empfohlen wird. Mit der manuellen Kipptechnik läßt sich zwar in der Routine ein hoher Probendurchsatz erzielen, was aber auf Kosten der Genauigkeit geht. Dagegen gestattet die manuelle Häkchentechnik eine sehr präzịse Messung, ist jedoch für hohen Probendurchsatz weniger gut geeignet. Gerinnungsanalysen werden heute überwiegend mit mechanisierten Geräten durchgeführt. Da bei bestimmten Reagenzien ein Einfluß der Meßtechnik auf den ISI nachgewiesen wurde (30), sollte bei verschiedenen Teilnehmern überprüft werden, ob ein gerätetechnischer Einfluß auf den ISI von Hepato Quick nachweisbar ist.

\subsection{Auswertung auf Basis "Prozent der Norm”}

Die Angabe des Resultats für die Thromboplastinzeit in „Prozent der Norm" weist gegenüber der "Ratio" eine Reihe von Vorteilen auf (27). Aus diesem Grund wurde von jedem Teilnehmer eine chargen- und gerätespezifische Bezugskurve auf der Basis eines einheitlichen Normalplasmapools für jede untersuchte Hepato QuickCharge erstellt. Die Auswertung in "Prozent der Norm” soll der Auswertung auf der Basis „Ratio" gegenübergestellt werden.

\section{Material und Methoden}

\subsection{Reagenzien und Geräte}

- Hepato Quick, Thromboplastinpräparat aus Primatenund Kaninchenhirn mit einem Zusatz von adsorbiertem Rinderoxalatplasma als Quelle für Fibrinogen und Faktor V sowie von Polybren als Heparininhibitor.

- WHO-Referenzthromboplastin OBT/79, Rinderhirnthromboplastin mit einem Zusatz von adsorbiertem Rinderoxalatplasma. OBT/79 ist ein von der WHO etablierter Sekundärstandard, der an dern 1967 eingeführten WHOStandard (Urstandard) mit der Bezeichnung 67/40 kalibriert wurde. Der ISI der Urstandards $67 / 40$ wurde definitionsgemäß gleich 1 gesetzt. In einer internationalen Multizenterstudie wurde der ISI von OBT/79 mit 1,011 ermittelt (9).

- Normalplasmapool (24).

- Hepato Quick-Control normal und abnormal (Boehringer Mannheim $\mathrm{GmbH}$ ).

- Calciumchloridlösung $3,2 \mathrm{mmol} / \mathrm{I}$ (Immuno AG, Wien).

- Calciumchlorid $0,01 \mathrm{~mol} / \mathrm{l}$ (Boehringer Mannheim $\mathrm{GmbH}$ ).

- Owrens Veronalpuffer-Lösung pH 7,35 (Boehringer Mannheim $\mathrm{GmbH}$ ). 
- Blutentnahme mit Sicherheits-Monovette ${ }^{\circledR}$ '. Charge Nr.05.1071; Natriumcitratlösung $0,106 \mathrm{~mol} / \mathrm{l}$ (Fa. Sarstedt, Nümbrecht).

- Die Bestimmung der Thromboplastinzeit erfolgte bei einem Teilnehmer mit der vorgeschriebenen manuellen Kippmethode, bei den anderen Teilnehmern entweder mit dem Kugelcoagulometer KC 10 oder dem Coagulometer nach Schnitger und Gross (Fa. Heinrich Amelung GmbH, Lemgo).

\subsection{Probengewinnung}

Nach erfolgter Venenpunktion wurde bis zur Markierung Blut aufgezogen (Verhältnis Blut/Antikoagulans = 9:1), durchgemischt und anschließend $10 \mathrm{~min}$ bei ca. $2000 \mathrm{~g}$ zentrifugiert. Das Plasma wurde sofort abpipettiert. und bis zur Bestimmung bei Raumtemperatur aufbewahrt. Der bei $-70^{\circ} \mathrm{C}$ gelagerte Normalplasmapool wurde bei $+37^{\circ} \mathrm{C}$ aufgetaut, um die kritische Phase der Kälteaktivierung möglichst schnell zu durchlaufen, und anschließend gut durchgemischt. Die lyophilisierten Plasmen wurden mit $0,5 \mathrm{ml}$ bidest. Wasser entsprechend der Vorschrift gelöst.

\subsection{Methoden}

Die Durchführung der einzelnen Methoden erfolgte entsprechend den Angaben der Hersteller:

\section{Hepato Quick, Methode mit Plasmaverdünnung}

Das Plasma wurde 1:10 mit Owrens Veronalpuffer verdünnt. $100 \mu \mathrm{l}$ dieser Plasmaverdünnung wurden mit $200 \mu$ l Hepato Quick-Reagenz vermischt und $2 \mathrm{~min}$ bei $37^{\circ} \mathrm{C}$ inkubiert. Danach wurden $100 \mu \mathrm{l}$ auf $37^{\circ} \mathrm{C}$ vortemperiertes 0,01 molares Calciumchlorid zugegeben und die Gerinnungszeit bestimmt.

\footnotetext{
Wz der Fa. Sarstedt, Nümbrecht.
}

WHO-Referenzthromboplastin OBT/79

2 Ampullen des Thromboplastins wurden mit je $2,2 \mathrm{ml}$ einer auf $37^{\circ} \mathrm{C}$ erwärmten $3,2 \mathrm{mmol} / \mathrm{I}$ Calciumchloridlösung rekonstituiert und anschließend zusammengegossen. $0,4 \mathrm{ml}$ Thromboplastin OBT/79 wurden $4 \mathrm{~min}$ bei $37^{\circ} \mathrm{C}$ inkubiert. Dann wurden $50 \mu$ l Plasma hinzupipettiert und die Gerinnungszeit bestimmt.

\section{Erstellung der Bezugskurven}

Zur Erstellung der Bezugskurven wurden mit Normalplasmapool folgende Verdünnungen mit physiologischer Natriumchloridlösung hergestellt: $100 \%, 50 \%$, 25\%, $12,5 \%$ und $6,25 \%$. Jede Verdünnungsstufe wurde mit der zu kalibrierenden Methode und dem internationalen Referenzthromboplastin OBT/79 in Vierfachmessung bestimmt.

\subsection{Auswertung und Qualitätskontrolle}

Der Ermittlung des ISI wurde das von Kirkwood (29) vorgeschlagene und von der WHO, ICSH und ICTH übernommene Modell (13) im Grundsätzlichen zugrunde gelegt und folgende Modifikationen vorgenommen:

1. Der mathematischen Beziehung zwischen INR und ISI (Gleichung 1) folgend, wurde nicht nur die Auftragung log sec (OBT/79) vs. log sec (Hepato Quick) zur Berechnung des ISI eingesetzt, sondern auch die Auftragung log ratio (OBT/79) vs log ratio (Hepato Quick). Diese Abweichung ist allerdings unerheblich, da in beiden Fällen die gleichen Resultate erhalten werden (s. Tab.4).

2. Anstelle der orthogonalen Regression (standardisierte Hauptkomponente) wurde zur Berechnung der Ausgleichsfunktion

$Y=a+b x$

auch das Verfahren nach Passing und Bablok $(5,25)$ herangezogen.

Tab. 1: Mittlerer, niedrigster und höchster Wert der Thromboplastinzeiten (in Klammern) der Normalplasmen. OBT/79 = Referenzthromboplastin; $H O=$ Hepato Quick; HS m. Verd. = Hausstandard Charge-Nr. 45326, Plasmamethode mit Verdünnung; HS o. Verd. = Hausstandard Charge-Nr. 45326, Plasmamethode ohne Verdünnung; Prüfch. m. Verd. = Produktionschargen Hepato Quick, Plasmamethode mit Verdünnung; Prüfch. o. Verd. = dgl., Plasmamethode ohne Verdünnung

\begin{tabular}{|c|c|c|c|c|c|c|}
\hline $\begin{array}{l}\text { Labor- } \\
\text { Nr. }\end{array}$ & & OBT/79 & $\begin{array}{l}\text { HO } \\
\text { HS m. Verd. }\end{array}$ & $\begin{array}{l}\text { HQ } \\
\text { HS o. Verd. }\end{array}$ & $\begin{array}{l}\text { HQ } \\
\text { Prüfch. m. Verd. }\end{array}$ & $\begin{array}{l}\text { HQ } \\
\text { Prüfch. o. Verd. }\end{array}$ \\
\hline 1 & $\begin{array}{l}\bar{x} \\
\text { TPZ-Bereich(s) } \\
\sigma \\
\sigma^{2}\end{array}$ & $\begin{array}{l}37,4 \\
33,1-43,0 \\
2,0 \\
4,1\end{array}$ & $\begin{array}{l}28,4 \\
24,3-34,2 \\
2,7 \\
7,1\end{array}$ & $\begin{array}{l}22,7 \\
19,8-27,2 \\
2,4 \\
5,9\end{array}$ & $\begin{array}{l}27,8 \\
23,4-32,9 \\
2,6 \\
6,5\end{array}$ & $\begin{array}{l}22,7 \\
19,8-26,7 \\
2,2 \\
5,0\end{array}$ \\
\hline 2 & $\begin{array}{l}\bar{x} \\
\text { TPZ-Bereich(s) } \\
\sigma \\
\sigma^{2}\end{array}$ & $\begin{array}{l}34,1 \\
30,2-37,2 \\
2,3 \\
5,5\end{array}$ & $\begin{array}{l}27,3 \\
23,7-30,2 \\
1,9 \\
3,6\end{array}$ & $\begin{array}{l}21,9 \\
19,3-25,2 \\
1,4 \\
2,1\end{array}$ & $\begin{array}{l}27,9 \\
24,3-30,1 \\
1,6 \\
2,7\end{array}$ & $\begin{array}{l}22,2 \\
19,6-24,4 \\
1,4 \\
1,9\end{array}$ \\
\hline 3 & $\begin{array}{l}\bar{x} \\
\text { TPZ-Bereich(s) } \\
\sigma \\
\sigma^{2}\end{array}$ & $\begin{array}{l}38,4 \\
33,3-41,3 \\
2,5 \\
6,5\end{array}$ & $\begin{array}{l}28,8 \\
25,5-33,5 \\
2,6 \\
6,6\end{array}$ & $\begin{array}{l}23,1 \\
21,0-26,5 \\
2,0 \\
3,8\end{array}$ & $\begin{array}{l}28,8 \\
25,5-33,0 \\
2,3 \\
5,4\end{array}$ & $\begin{array}{l}22,8 \\
20,0-26,0 \\
1,8 \\
3,4\end{array}$ \\
\hline 4 & $\begin{array}{l}\bar{x} \\
\text { TPZ-Bereich(s) } \\
\sigma \\
\sigma^{2}\end{array}$ & $\begin{array}{l}35,0 \\
31,7-41,1 \\
2,6 \\
6,9\end{array}$ & $\begin{array}{l}26,7 \\
23,9-32,3 \\
2,3 \\
5,1\end{array}$ & $\begin{array}{l}21,9 \\
19,4-25,6 \\
1,7 \\
3,0\end{array}$ & $\begin{array}{l}27,5 \\
23,9-32,8 \\
2,4 \\
5,9\end{array}$ & $\begin{array}{l}22,4 \\
19,2-26,1 \\
1,8 \\
3,3\end{array}$ \\
\hline 5 & $\begin{array}{l}\bar{x} \\
\text { TPZ-Bereich(s) } \\
\sigma \\
\sigma^{2}\end{array}$ & $\begin{array}{l}36,3 \\
28,0-39,3 \\
2,9 \\
8,2\end{array}$ & $\begin{array}{l}29,5 \\
24,9-32,8 \\
2,7 \\
7,1\end{array}$ & $\begin{array}{l}23,0 \\
20,5-25,5 \\
1,9 \\
3,5\end{array}$ & $\begin{array}{l}28,6 \\
24,1-31,9 \\
2,7 \\
7,5\end{array}$ & $\begin{array}{l}22,7 \\
19,6-25,5 \\
1,8 \\
3,3\end{array}$ \\
\hline
\end{tabular}



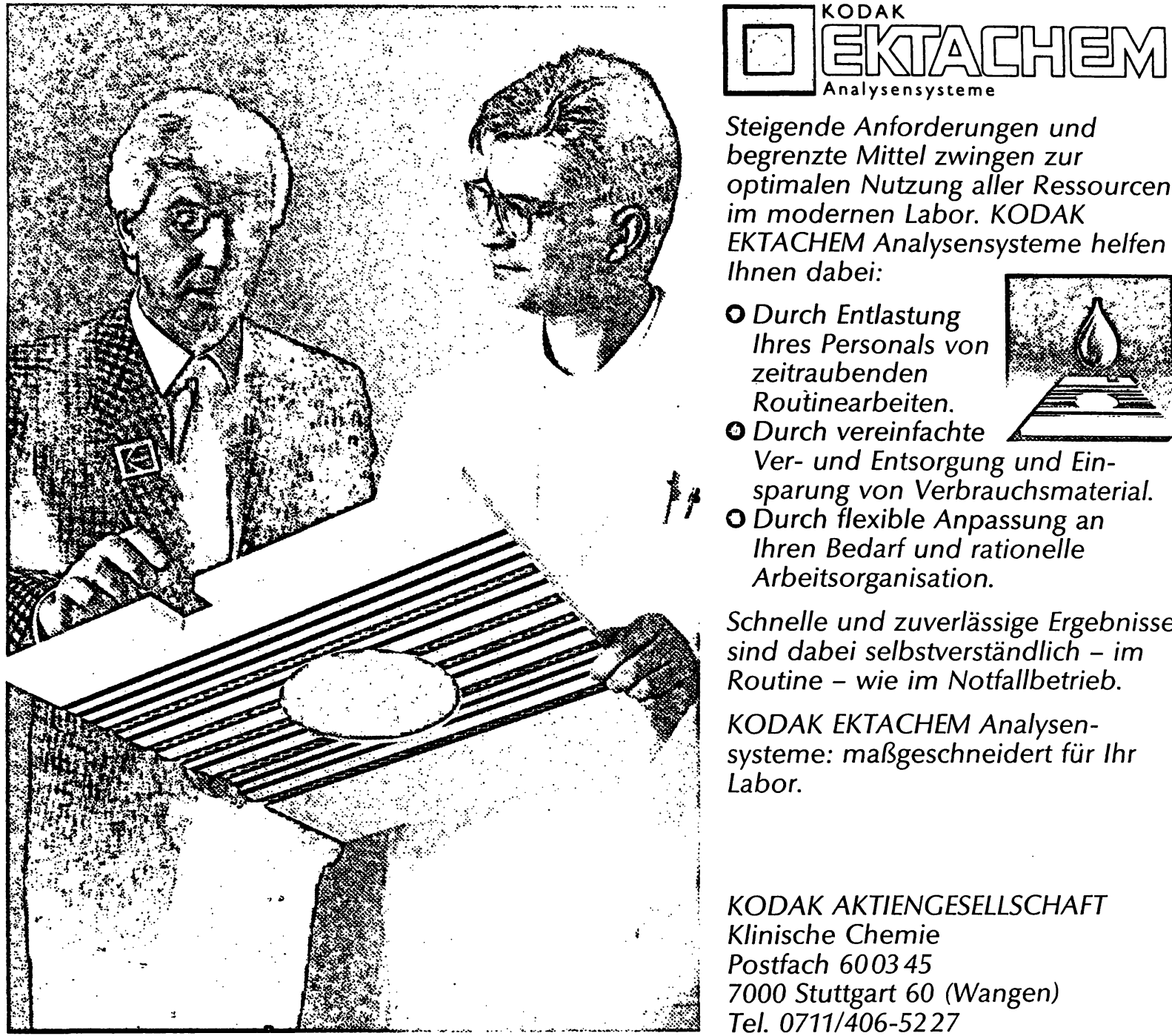

Steigende Anforderungen und begrenzte Mittel zwingen zur optimalen Nutzung aller Ressourcen im modernen Labor. KODAK

EKTACHEM Analysensysteme helfen Ihnen dabei:

- Durch Entlastung Ihres Personals von zeitraubenden Routinearbeiten.

- Durch vereinfachte

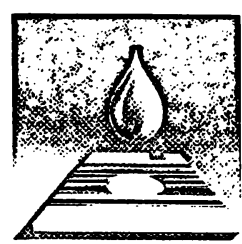
Ver- und Entsorgung und Ein-

sparung von Verbrauchsmaterial. - Durch flexible Anpassung an Ihren Bedarf und rationelle Arbeitsorganisation.

Schnelle und zuverlässige Ergebnisse sind dabei selbstverständlich - im Routine - wie im Notfallbetrieb.

KODAK EKTACHEM Analysensysteme: maßgeschneidert für Ihr Labor.

KODAK AKTIENGESELLSCHAFT Klinische Chemie Postfach 600345 7000 Stuttgart 60 (Wangen) Tel. 0711/406-5227

Rufen Sie uns an oder besuchen Sie uns auf der MEDICA in Halle 4, Stand 4H 06
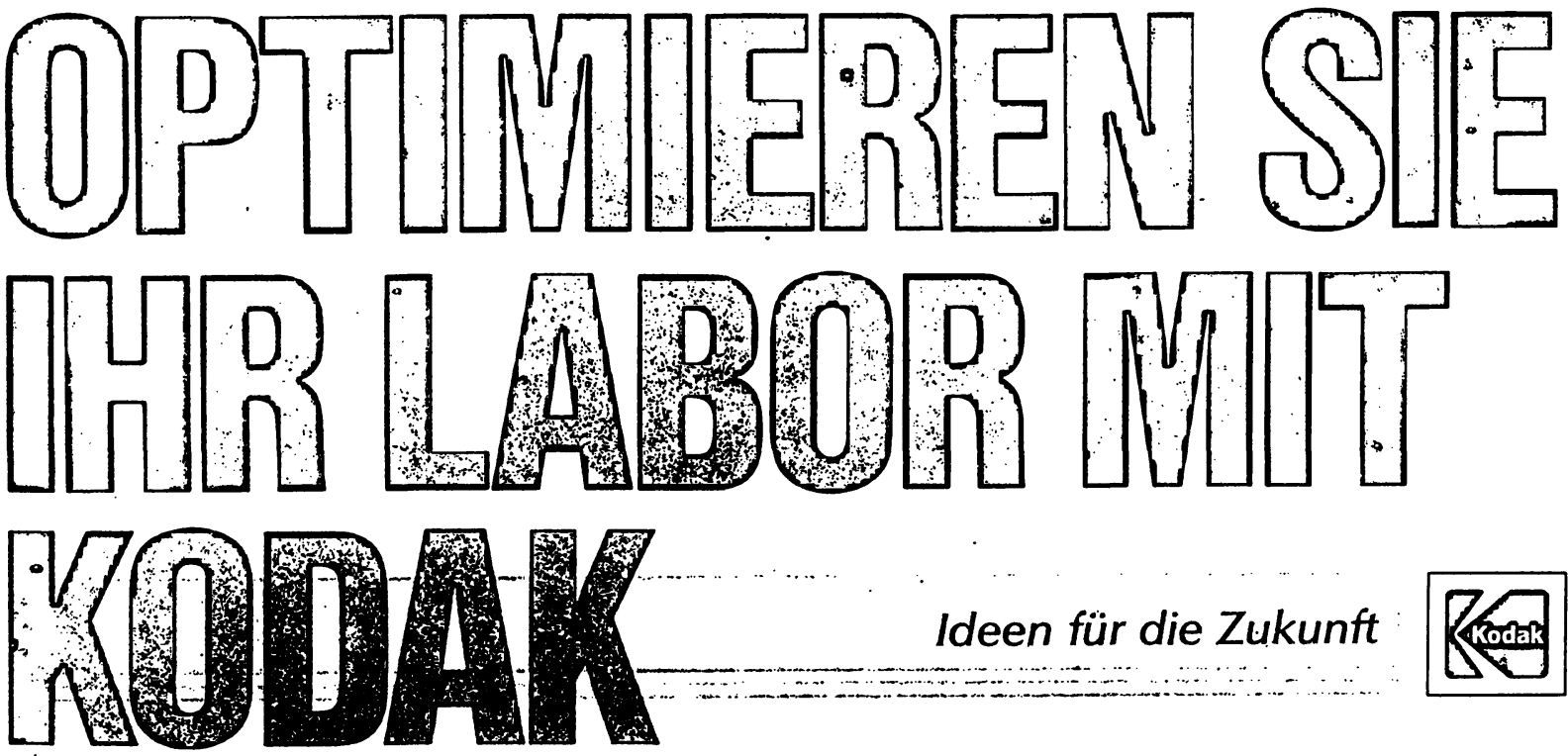

Ideen für die Zukunft

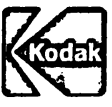




\section{DER Hämafologie- Analysafor 8702}

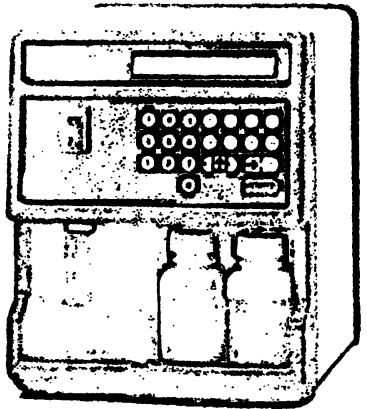

- Analysator für 6 Meßparameter

- PLT ohne Vorbereitung aus Vollblut - Mikroprozessor-Technik für Sicherheit

- Selbsthilfeprogramm bei Störungen - MedGV

Fragen Sie uns nach optimalen Systemen auch für Ihr Labor!
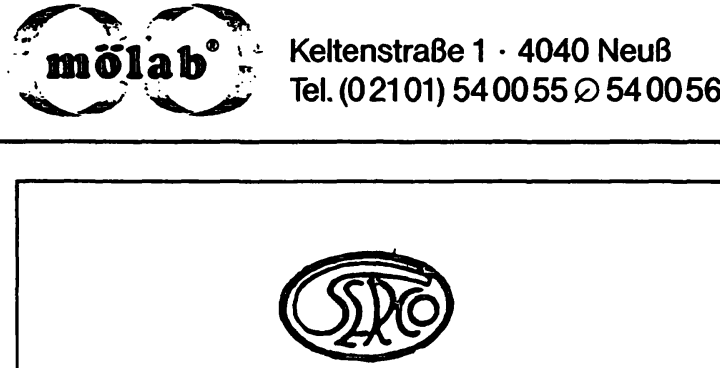

Serologisch-Chemisches Institut

Dr. E. Cohnen,

Wilhelm-Levison-Straße 5

5300 Bonn 1

Telefon (0228) 215306

36 Jahre Blutgruppen-Testseren „Problemlose Blutgruppentestung mit der SERCO-Spezial-Tüpfelplatte“

Anti-A/B/AB/A $/ \mathrm{H} / \mathrm{A}_{\mathrm{HP}} / \mathrm{D} / \mathrm{C} / \mathrm{c} / \mathrm{E} / \mathrm{e} /$ $\mathrm{C}^{\mathrm{W}} / \mathrm{M} / \mathrm{N} / \mathrm{S} / \mathrm{s} / \mathrm{K} / \mathrm{k} / \mathrm{P} / \mathrm{Fy}^{\mathrm{a}} / \mathrm{Fy}^{\mathrm{b}} / \mathrm{Jk}^{\mathrm{a}} /$

$\mathrm{Jk}^{\mathrm{b}} / \mathrm{Le}^{\mathrm{a}} / \mathrm{Le}^{\mathrm{b}} / \mathrm{Lu}^{\mathrm{a}} / \mathrm{Lu}^{\mathrm{b}} / \mathrm{G}^{\mathrm{c}} / \mathrm{Gm}^{1} / \mathrm{Gm}^{2}$ $\mathrm{Gm}^{3} / \mathrm{Gm}^{5} / \mathrm{Gm}^{17} / \mathrm{Gm}^{21} / \mathrm{Km}^{1} / \mathrm{Km}^{3}$
Der Bunsenbrenner für Mikrobiologen

Beide Hande frei for's Experiment und mil dem FuB Gas geben

Touch-o-matik mit

Ped-o-mat

Fur Handbedienung ohne Umschalten bereit; flache Konstruktion for ermodungsireies Arbeiten. Robust, weder Batterle noch Netzanschluß notig.

FuBbedienung PED-O-MAT laBt sich auch nachrusten. Touch-0-matic einfach 1 Woche einsenden.

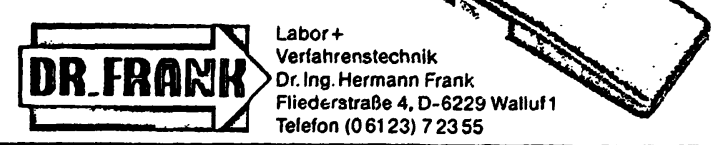

\section{Torkate}

1 Gefriermikrotom (Jung 1206) mit Objektund Messerkühlung,

- 2 Schlittenmikrotome (Jung HN 40) und

- 2 elektr. Kühlplatten zu verkaufen.

Telefon (071 71) 68110

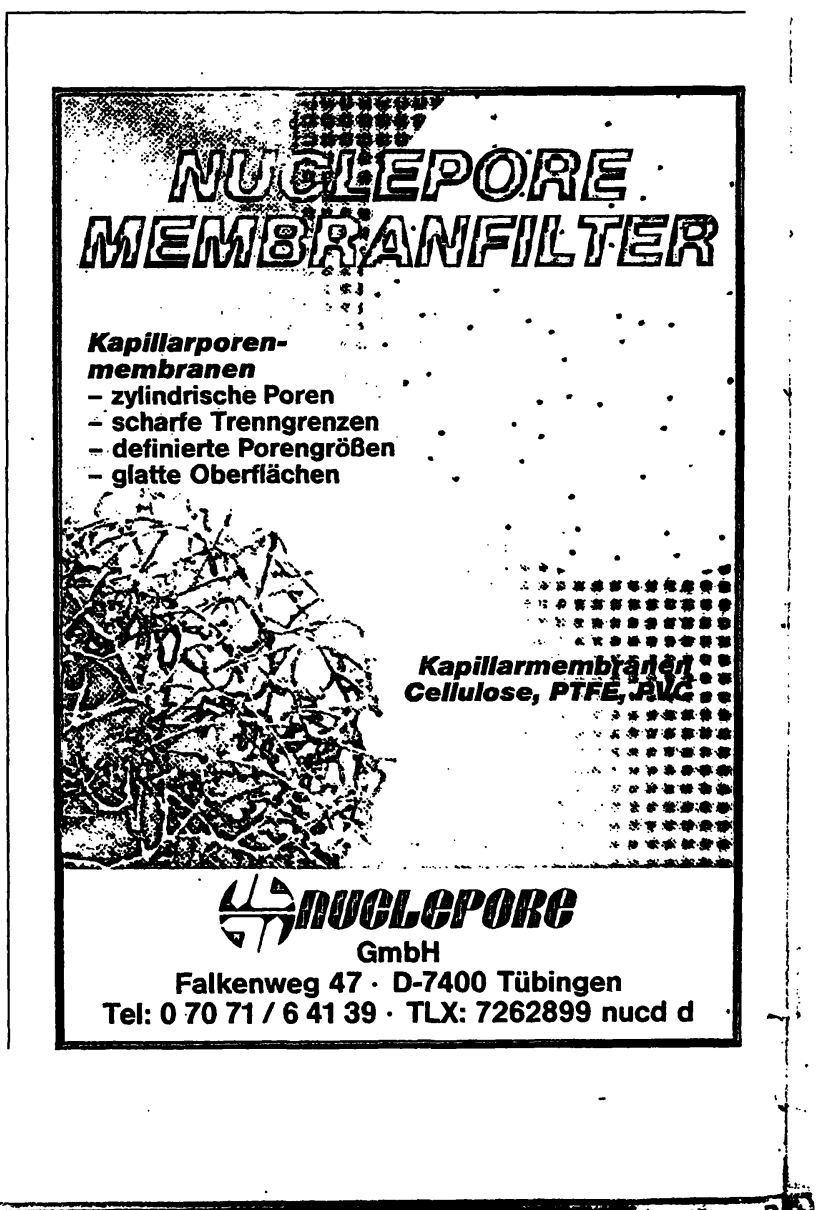


Vor der Studie wurde von jedem Teilnehmer die Präzision in der Serie bestimmt. Dazu wurden an einem Kontrollplasma 20 Werte nacheinander gemessen. Daraus ergaben sich für die Präzision in der Serie auf Basis von Sekunden folgende Variationskoeffizienten OBT/79= 1.3-3,8\%; Hepato Quick $=0,8-1,8 \%$. Diese Messungen wurden am Ende der Studie wiederholt. Die Variationskoeffizienten lagen in der gleichen Größenordnung.

\section{Ergebnisse}

\subsection{Streuung der Thromboplastinzeiten (TPZ) der Normalprobanden}

An jeweils 10 Tagen wurden von jedem Teilnehmer die Thromboplastinzeiten von je 2 gerinnungsnormalen Probanden mit OBT/79 und den zu prüfenden Hepato QuickChargen bestimmt. Tab. 1 enthält den Mittelwert, die niedrigste und die höchste gemessene Thromboplastinzeit,

Tab. 2: Empfehlungen zur Intensität der oralen Antikoagulation bei verschiedenen Indikationen (15) .

\begin{tabular}{|c|c|c|}
\hline \multirow[t]{2}{*}{ Indikation } & \multicolumn{2}{|l|}{ INR } \\
\hline & Zielwert & Bereich \\
\hline $\begin{array}{l}\text { Prä- und perioperative orale } \\
\text { Antikoagulation, Beginn zwei } \\
\text { Wochen vor einer Operation } \\
\text { - Hüftgelenksoperationen } \\
\text { - Andere Operationen }\end{array}$ & $\begin{array}{l}2,5 \\
2,0\end{array}$ & $\begin{array}{l}2-3 \\
1,5-2,5\end{array}$ \\
\hline $\begin{array}{l}\text { Primär- und Sekundärprävention } \\
\text { venöser Thrombosen }\end{array}$ & 2,5 & $2-3$ \\
\hline $\begin{array}{l}\text { Manifeste venöse Thrombosen, } \\
\text { Lungenembolien, Verhinderung } \\
\text { rezidivierender venöser Thrombosen }\end{array}$ & 3 & $2-4$ \\
\hline $\begin{array}{l}\text { Prävention von arteriellen } \\
\text { Thromboembolien einschließlich } \\
\text { Patienten mit künstlichen } \\
\text { Herzklappen }\end{array}$ & 3,5 & $3-4,5$ \\
\hline
\end{tabular}

Tab. 3: Verteilung der Patienten nach den INR-Werten von OBT/ 79 und Hausstandard Hepato Quick (HQ)

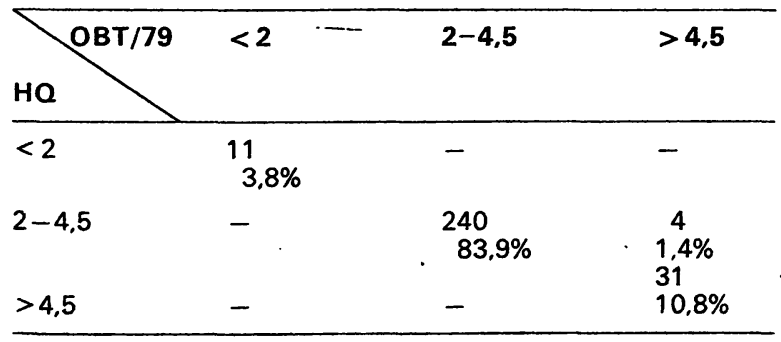

$N=286$ die Standardabweichung und die Varianzen. Bei der Bewertung der Varianz ist zu beachten, daß nicht die Präzision in der Serie der jeweiligen Methode angegeben wird, sondern die Varianz der Thromboplastinzeiten innerhalb der einzelnen Probandenkollektive. Diese ist naturgemäß relativ hoch, da es sich um unterschiedliche Normalplasmen handelt. Betrachtet man dagegen die Varianz der Thromboplastinzeiten des Normalplasmapools, der für alle Teilnehmer gleich war, dann ergeben sich Variationskoeffizienten von $1,4 \%$ bis $1,9 \%$.

\subsection{Intensität der Antikoagulation der einbezogenen Patienten}

Für die Einstellung der oralen Antikoagulantien-Patienten anhand der INR werden für die verschiedenen Indikationen unterschiedliche therapeutische Bereiche vorgeschlagen (s. Tab. 2).

Tab. 3 gibt einen Überblick über die Einstellung der in die Studie aufgenommenen Patienten anhand einer Einteilung auf die drei Bereiche < 2 INR; 2-4,5 INR und $>4,5$ INR nach dem internationalen Referenzthromboplastin gegen die INR des Hausstandards in einem Neunfelderdiagramm. 83,9\% der Patienten liegen nach beiden Methoden in dem für die orale Antikoagulation empfohlenen therapeutischen Bereich von INR 2 bis 4,5. 3,8\% werden als unterdosiert (INR $<2,0$ ) und $10,8 \%$ als überdosiert (INR > 4,5) ausgewiesen. Die Übereinstimmung der Eingruppierung zwischen Hepato Quick und OBT/79 beträgt $98,6 \%$. Lediglich bei 4 Patienten $(1,4 \%)$ lag der Hepato Quick-Wert noch im therapeutischen Bereich, während mit OBT/79 Werte über INR 4,5 erhalten wurden.

\subsection{International Sensitivity Index (ISI) von Hepato Quick}

Für Hepato Quick wurde der ISI eines Hausstandards durch parallele Kalibrierung der dafür vorgesehenen $\mathrm{He}$ pato Quick-Charge bei allen fünf Teilnehmern sowie die ISI-Werte von fünf verschiedenen Produktionschargen ermittelt.

\subsubsection{Hausstandard, Hepato Quick-Charge Nr.696883-01}

Abb. 1 enthält die von allen Teilnehmern bei der Kalibrierung mit dem Hepato Quick-Hausstandard an insgesamt 100 Normalprobanden und 284 stabil eingestellten oral antikoagulierten Patienten gegen die mit dem internationalen Referenzthromboplastin OBT/79 gemessenen Werte. Es wurden die sec, die log sec- und die log ratioWerte gegeneinander aufgetragen. Zur Berechnung der Ratio (PR) nach der Gleichung

$P R=\frac{T P Z_{\text {Patient }}[\mathrm{sec}]}{T P Z_{\text {Normal }}[\mathrm{sec}]}$

Tab. 4: Kalibrierung der Hepato Quick-Hausstandard-Charge Nr. $696883-01$ (Plasmamethode mit Verdünnung) gegen das WHOReferenzthromboplastin OBT/79. Berechnung aufgrund der gesamten Meßwerte von 100 Normalprobanden und 284 oral antikoagulierten Patienten

\begin{tabular}{|c|c|c|c|c|c|c|c|}
\hline \multirow[t]{2}{*}{ Darsteliung } & \multicolumn{2}{|c|}{ orthogonale Regression } & & \multicolumn{2}{|c|}{ Bablok/Passing } & \multirow[t]{2}{*}{ ISI } & \multirow{2}{*}{$\begin{array}{l}\text { Korrelationsk } \\
\text { oeffizient }\end{array}$} \\
\hline & $\mathbf{a}$ & b & . & a. & b & & \\
\hline sec vs. sec & -1.187 & 0,968 & & 1,136 & 0,988 & . & 0,968 \\
\hline $\log \sec$ vs. $\log \sec$ & 0,190 & 0,938 & & 0.172 & 0,944 & 0,95 & 0,982 \\
\hline log ratio vs. log ratio & $-0,009$ & 0,938 & & $-0,014$ & 0,944 & & 0.982 \\
\hline
\end{tabular}



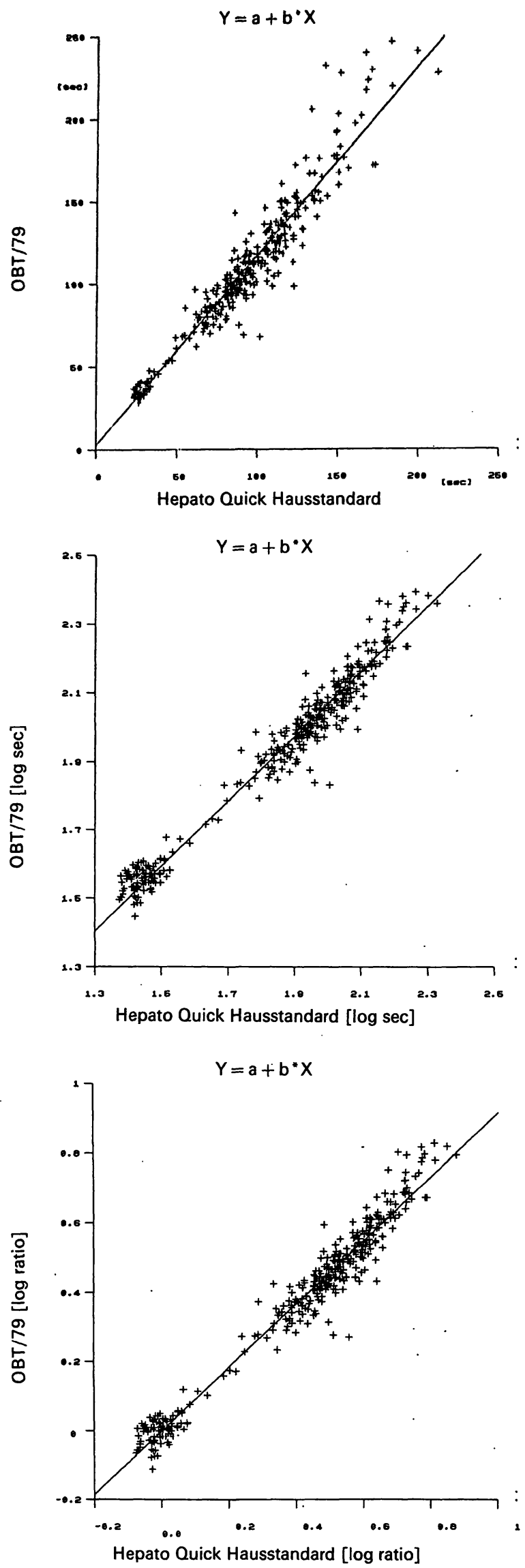

Abb. 1: Kalibrierung des Hepato Quick-Hausstandards gegen das internationale Referenzthromboplastin OBT/79 (Ergebnisse sämtlicher Teilnehmer)

wurde für $T P Z_{\text {Normal }}$ der Mitttelwert aus allen Werten der Normalprobanden eingesetzt. Dieser beträgt für OBT/79 36,18947 sec und für den Hepato Quick-Hausstandard (Plasmamethode mit Verdünnung) $28,16842 \mathrm{sec}$. Bei jeder Auftragung ergibt sich eine gemeinsame Ausgleichgerade, sowohl für die Werte der Normalprobanden als auch für die Patientenwerte, die jedoch geringfügige rechnerische Abweichungen von der Linearität (Cusumtest) aufweisen.

Ursache hierfür scheint die Tatsache zu sein, daß mit OBT bei niedrigen Quick-Werten relativ stark verlängerte Zeiten erhalten werden. Dies führt, wie aus Abb.1 a ersichtlich, im oberen Bereich zur Abweichung von der Linearität.

\subsubsection{Hepato Quick, Produktionschargen}

Die von den einzelnen Teilnehmern durchgeführten Kalibrierungen der fünf Produktionschargen sind in Abb. 2 zusammengestellt. Wie in Abb. 1 wurden die mit den einzelnen Chargen gemessenen Werte gegen die OBT-Werte als log ratio aufgetragen. Die daraus resultierenden ISIWerte sind in Tab. 6 aufgelistet.

\subsubsection{Kalibrierung der Produktionschargen gegen den Hausstandard Hepato Quick}

In Abb. 3 sind für jeden Teilnehmer die mit der jeweiligen Produktionscharge gegen die mit dem Hausstandard gemessenen Werte als log ratio aufgetragen. Es wurde jeweils die entsprechende Regressionsgerade berechnet und dadurch der ISI der Produktionschargen durch Kalibrierung gegen den Hausstandard erhalten. Abweichungen von der Linearität gemäß Cusumtest treten in keinem Fall auf. Eine Zusammenstellung der ISI-Werte findet sich in Tab. 6.

\subsubsection{Auswertung in Prozent der Norm}

Parallel zu der Auswertung auf der Basis von Sekunden oder Ratio wurden die Werte auch in Prozent der Norm verglichen. Dazu wurde von jedem Teilnehmer eine Bezugskurve mit einem einheitlichen Normalplasmapool erstellt (siehe 4.3). An dieser Bezugskurve wurden die Werte für die einzelnen Patienten und Probanden in Prozent der Norm ermittelt.

In Abb. 4 sind die von allen Teilnehmern mit dem Hausstandard Hepato Quick gegen die mit OBT/79 erhaltenen Werte als Prozent der Norm aufgetragen. Es zeigt sich, daß auch bei dieser Vorgehensweise ein linearer Zusammenhang zwischen dem zu kalibrierenden Reagenz und dem internationalen Referenzthromboplastin besteht und die Werte ineinander umgerechnet werden können.

Der Beziehung $\mathrm{PR}^{\mid \mathbf{I S}}=\mathbf{I N R}$

entspricht somit die Gleichung

$\frac{(\% \text { der Norm })_{\text {HO }}}{A}-B=(\% \text { der Norm })_{O B}$.

Für den Hausstandard Hepato Quick gilt:

$A=1,568$

$B=-0,73$. 


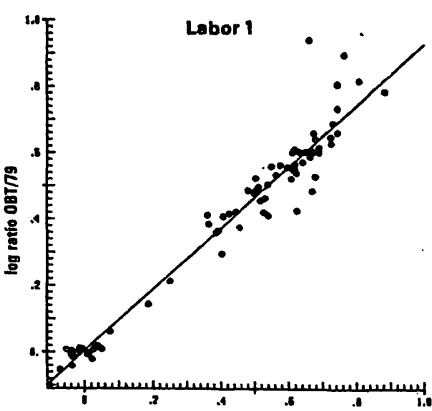

log ratio Hepato Quick Charge 698848-01 (Produktionscharge)

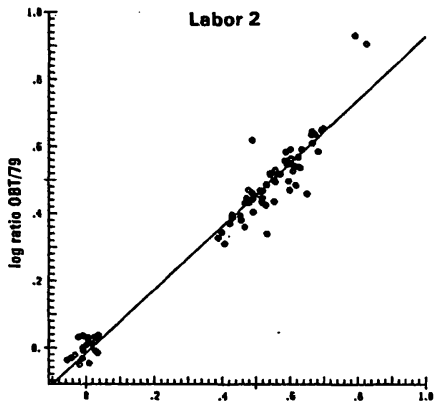

log ratio Hepato Quick Charge 696819-01 (Produktionscharpe)

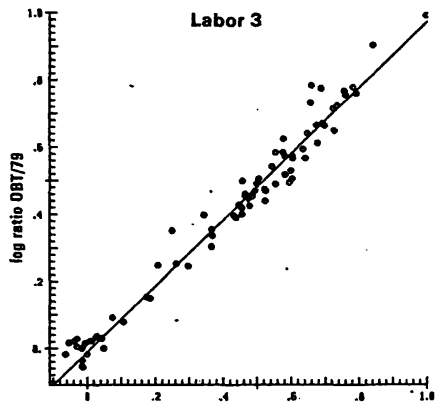

log ratio Hepato Quick Charge 696864-01 (Produktionscharge)

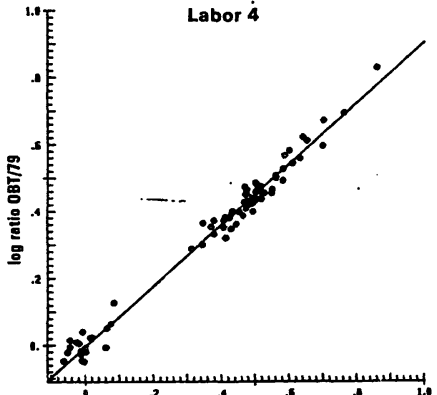

log metio Hepato Quick Charge 696820-01 (Produkionscharge)

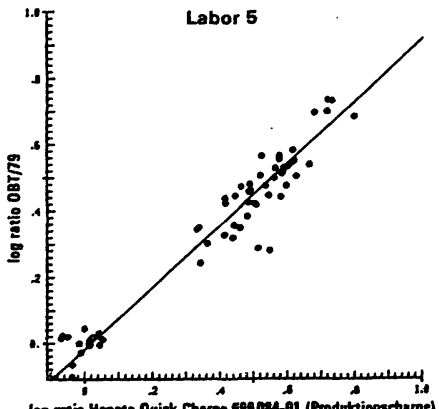

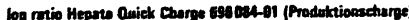

Abb. 2: Kalibrierung von 5 Hepato QuickReagenzchargen gegen das internationale Referenzthromboplastin OBT/79
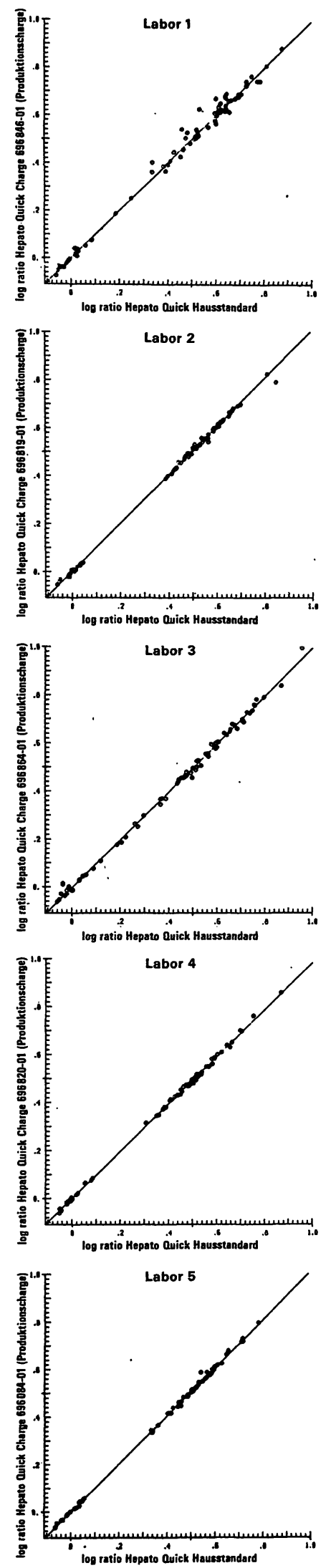

Abb. 3: Kalibrierung von 5 Hepato Quick Reagenzchargen gegen den Hepato

Quick-Hausstandard

Lab.med. 12: 395 (1988) 
Tab. 5: Ergebnisse der Kalibrierung der Hepsto Quick-Hausstandard. Charge Nr. 696883.01 (Plasmamethode mit Verdünnung) gegen das WHO-Referenzthromboplastin in 5 verschiodenen Labors ( $=$ Anzahl der Normalprobanden + oral antikoagulierte Patienten)

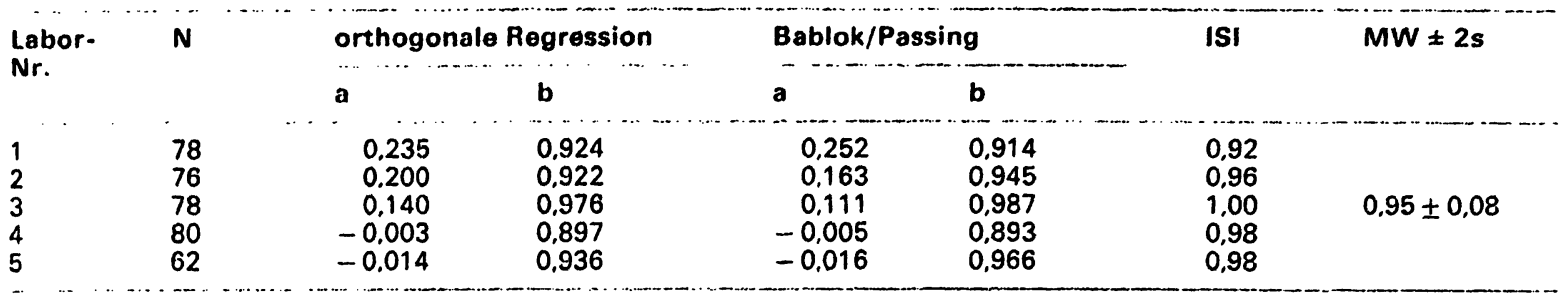

Tab. 6: International Sensitivity Index (ISI) von 5 verschiedenen Produktionschargen Hepato Quick

\begin{tabular}{llllll}
$\begin{array}{l}\text { Labor- } \\
\text { Nr. }\end{array}$ & N & a & b & ISI & MW \pm 2 s \\
\hline 1 & 78 & 0,257 & 0,915 & 0,93 & \\
2 & 76 & 0,173 & 0,936 & 0,95 & \\
3 & 78 & 0,132 & 0,979 & 0,99 & $0,95 \pm 0,054$ \\
4 & 80 & 0,229 & 0,913 & 0,92 & \\
$\mathbf{5}$ & 62 & 0,020 & 0,938 & 0,95 & \\
\hline
\end{tabular}

\section{Diskussion}

Im Rahmen einer multizentrischen Studie wurden insgesamt 6 verschiedene Chargen von Hepato Quick. nach dem WHO-Modell gegen das internationale Referenzthromboplastin OBT/79 kalibriert. Da das internationale Referenzthromboplastin nicht in beliebiger Menge zur Verfügung steht, soll für die routinemäßige Kalibrierung der Reagenzchargen aus der laufenden Produktion von jedem Reagenzienhersteller ein eigens etablierter Hausstandard verwendet werden. Deshalb wurde von den beteiligten Laboratorien jeweils eine einheitliche Reagenzcharge, die als Hausstandard etabliert werden soll, und zusätzlich eine zweite, in jedem Labor verschiedene Charge dem Kalibrierverfahren unterworfen.

Für die als Hausstandard vorgesehene Hepato QuickReagenzcharge wurde ein mittlerer ISI von 0,95 ermittelt. Die Varianz des ISI von Labor zu Labor hält sich in engen Grenzen (VK $=4,0 \% ; 2 s= \pm 0,08$ ). Dies zeigt, daß in allen beteiligten Laboratorien mit hoher Zuverlässigkeit gearbeitet wurde. Da eine einheitliche Meßtechnik bewußt nicht festgelegt war, wurde neben der im WHOModell empfohlenen „Kipp-Methode” sowohl das Koagulometer nach Schnitger und Groß als auch das Kugelkoagulometer KC 10 der Fa. Amelung verwendet. Es ist hervorzuheben, daß dadurch keine Unterschiede aufgetreten sind, obwohl die „Kipp-Technik” als problematisch anzusehen ist. Zumindest für ein nach Owren modifiziertes Reagenz wie Hepato Quick ist daher keine Geräteabhängigkeit des ISI - im Gegensatz zu den herkömmlichen Thromboplastinreagenzien. (30) - zu erwarten.

Weiterhin war zu prüfen, ob die Kalibrierung mit dem Hausstandard zum gleichen Ergebnis führt wie die Kalibrierung mit dem internationalen Referenzthromboplastin. Zu diesem Zweck wurde der ISI der verschiedenen Produktionschargen einmal mit dem Referenzthromboplastin OBT/79 und einmal mit dem Hausstandard Hepato Quick berechnet. Wie aus Tab. 6 zu ersehen ist, werden dabei befriedigend übereinstimmende Werte erhalten. Damit ist gezeigt, daß die Kalibrierung mit dem Hausstandard prinzipiell ebenso möglich ist wie mit dem internationalen Referenzthromboplastin.
Für die fünf Hepato Quick-Produktionschargen wurden $|S|$-Werte zwischen 0,92 und 0,99 erhalten, mit einem mittleren ISI von 0,95. Die Varianz des ISI von Charge zu Charge bewegt sich mit einem VK von $3,5 \%$ und einem $2 s$-Bereich von $\pm 0,054$ in der gleichen Größenordnung wie die Varianz des ISI von Labor zu Labor. Diese gute Chargenkonstanz, die aufgrund des Prinzips von Hepato Quick durchaus zu erwarten war, sollte durch weitere Untersuchungen, wie sie im Rahmen der routinemäßigen Kalibrierung neu produzierter Reagenzchargen sowieso vorgesehen sind, zusätzlich untermauert und beibehalten werden. Sie bietet unter Berücksichtigung der empfohlenen therapeutischen Bereiche für die orale Antikoagulation auf der Basis der International Normalized Ratio (INR) und der Tatsache, daß der ISI für Hepato Quick mit ca. 0,95 Schwankungen in den Endergebnissen vermindert, sogar die Möglichkeit, für Hepato Quick einen einheitlichen, chargenunabhängigen ISI zu verwenden. Dies würde eine erhebliche Vereinfachung bedeuten, insbesondere weil dadurch Verwechslungsfehler ausgeschlossen werden.

Geht man von den empfohlenen Bereichen für eine optimale Einstellung der Antikoagulantienpatienten von INR 2,0 bis 4,5 je nach Indikation aus, dann zeigt sich, daß $84 \%$ der Patienten sowohl mit dem internationalen Referenzthromboplastin OBT/79 als auch mit Hepato Quick in diesem Bereich liegen. Von den 46 (16\%) außerhalb dieses Bereiches liegenden Patienten werden 42 $(14,6 \%)$ mit beiden Reagenzien gleich und nur 4 von 286 Patienten $(1,4 \%)$ werden mit Hepato Quick in die Gruppe INR $<4,5$ und mit OBT/79 in den Bereich INR $>4,5$

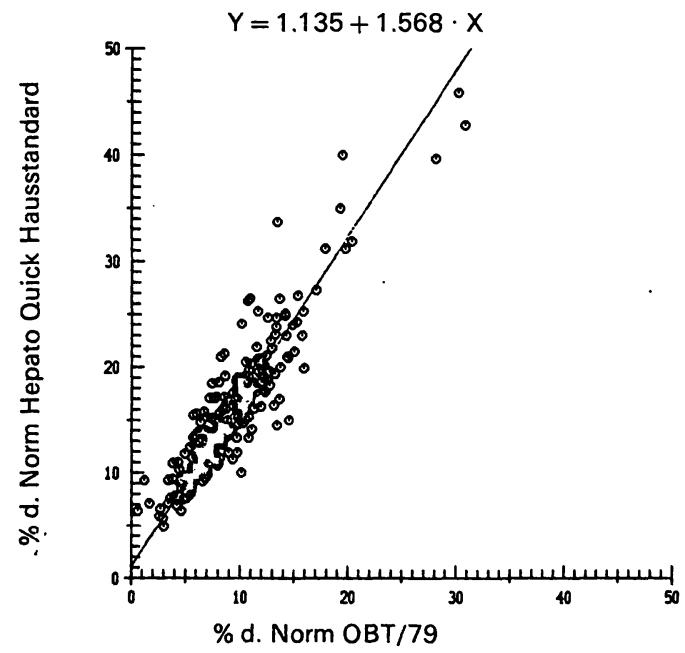

Abb. 4: Vergleich der Thromboplastinzeitwerte von 286 Patienten umgerechnet in Prozent der Norm des Hepato Quick-Hausstandards und des internationalen Referenzthromboplastins OBT/7j 


\section{INTELLIGENZ}

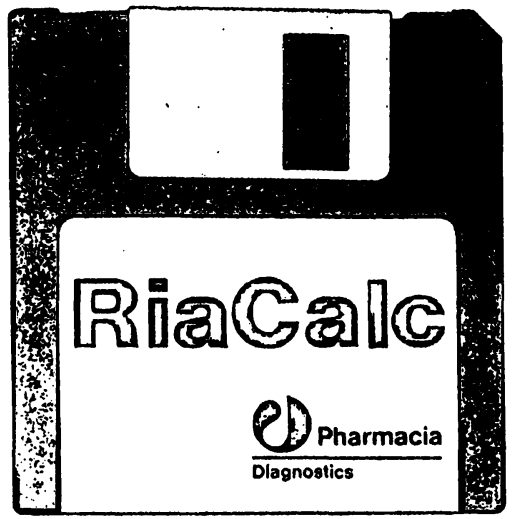

Die Qualitätskontrolle im Labor, die Verwaltung von Probendaten, die $\Lambda$ utomation der $\Lambda$ ssays erfordern intelligente Programme; RI $\Lambda$ Calc,

FIACalc, W-Base und Phamas ermöglichen umfassendes und zeitgemäßes Labormanagement.

Intelligenz im Labor heißt, mit uns Systeme verknüpfen - individuelle Lösungen entwickcln.

\section{$\mathbb{P R A Z I S I O N ~}$}

Genauigkeit, Reproduzierbarkeit und Flexibilität sind Maßstäbe, an denen sich ein Testkit messen lassen muß.

Mit Coated-tubes, Delfia und RAST ${ }^{\circledR}$ werden diesc Anforderungen erfüllt.

Präzision ist auch in Zukunft für uns eine sellsstverständliche Verpflichtung.

Diese Präzision wird mit Intelligenz und High-Tech zukunftsweisend zu ökonomischen Cesamtlösungen führen.

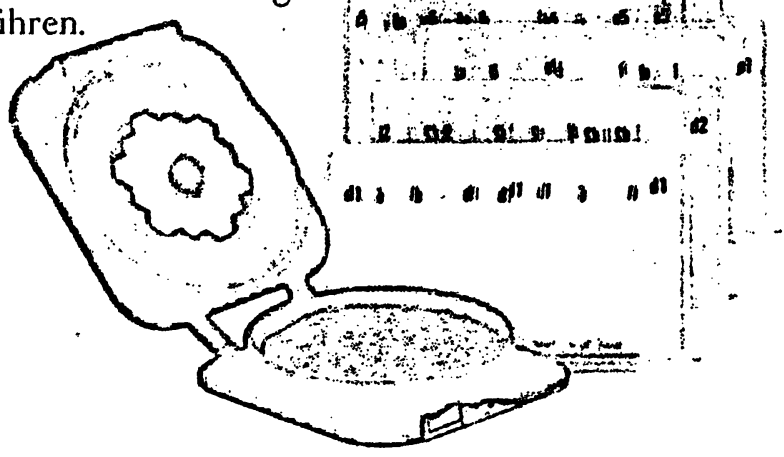

\section{AUF EINE GUTE ZUSAMMENARBEIT.}

Ökonomischer und ökologischer Einsatz von Geräten in Routine und Forschung ist heute wichtiger denn je.

Beispiele dafür sind Camma- und BetaCounter, Luminometer und Fluorometer. High-Tech bedeutet die kontinuierliche Weiterent wicklung kompletter Systemlösungen. Der Weg ist vorgezcichnet: Delfia, Betal'late...

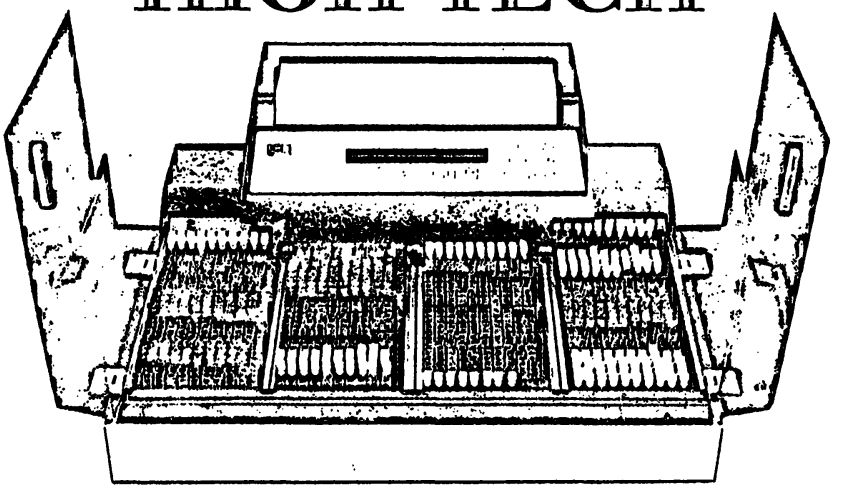

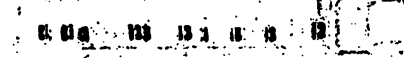

(1)

I

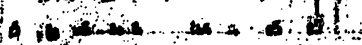




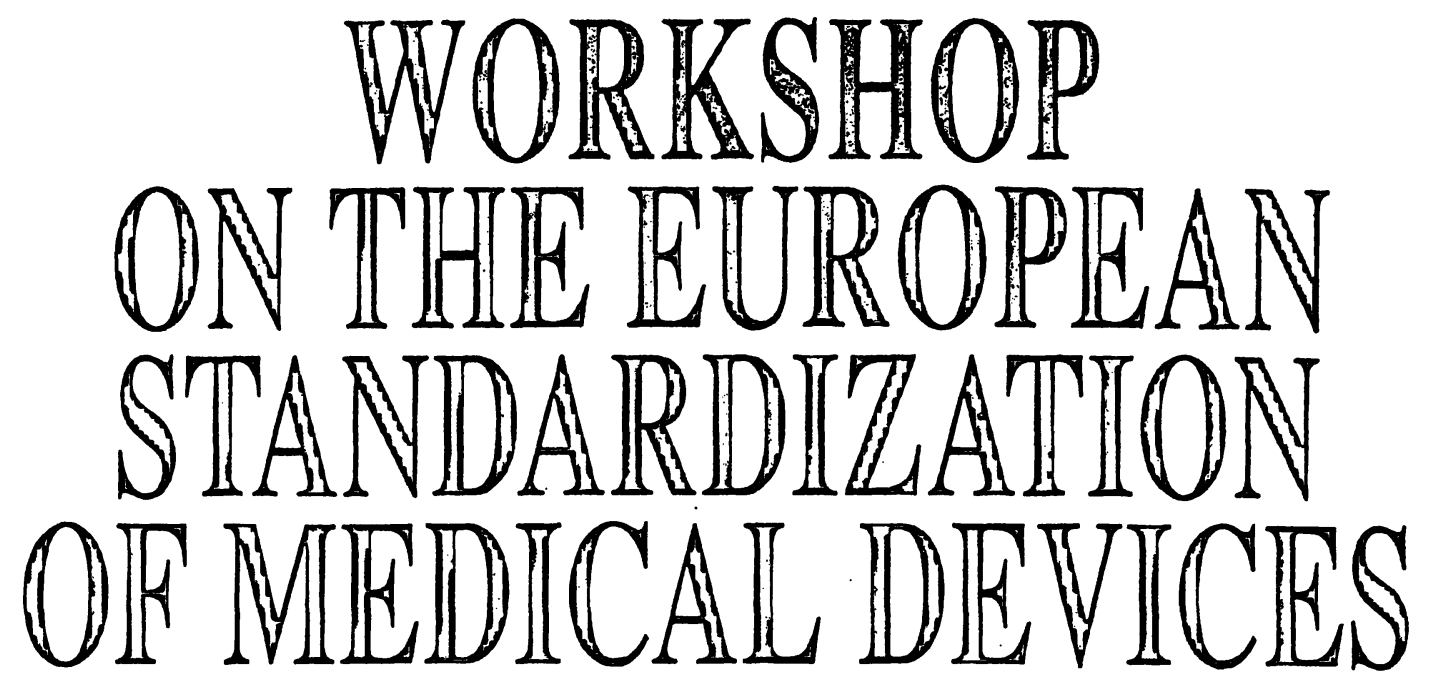

\section{BRUSSELS \\ SHERATON HOTEL \\ 12 - 13 - 14 DECEMBER 1988}

\section{FIRST ANNOUNCEMENT}

\section{PRELIMINARY PROGRAMME}

After an overall presentation by the Commission of the European Communities of the current and foreseen European legislation on medical devices in the context of the "New Approach", topics such as legal aspects, standstill agreement, agreements with the European Free Trade Association, advanced informatics in medecine, electromagnetic compatibility will be explained.

An introduction to the working procedures within CEN and CENELEC will be given and such aspects as the agreements between ISO/IEC and CEN/CENELEC, the relations with European and Intemational associations, and certification will also be treated.

General sessions will deal with horizontal activities such as quality assurance. biological safety and biocompatibility, freedom from contamination. connector compatibility, clinical evaluation, ...

The participants will be divided later into several working groups dealing with specific products such as surgical instruments, single use devices. non-powered implants, powered implants, electromedical devices, sutures, surgical dressings, drapes. gloves and gowns, prosthetics and orthotics. catheters and cannulae, transfusion, infusion and dialysis devices. syringes and needles, dental products and equipment. ophthalmic optics, clinical laboratory devices and practices, in-vitro diagnostics. informatic processing and communication,...

These sessions are aimed at defining or confirming the European standardization priorities in the different medical areas and.in particular, at responding to the need created by the forthcoming medical legislation in Europe.

This workshop is open to all persons, companies. governmental agencies, associations of manufacturers, suppliers. users, consumers, professional and trade associations involved in the medical devices field.

\section{INFORMATION - REGISTRATION}

Please contact Roland GERARD at CEN

2. rue Bréderode

1000 - Bruxelles

Belgium

Phone: (32) - 2 - 51968 I1

Fax: (32) - 2 - 5196819

Telex: 26257 cenlec b

Teletex: $206-2210097=$ CENCEL

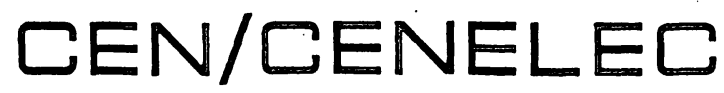


eingestuft. Hierbei ist allerdings zu beachten, daß diese Abweichungen innerhalb der Schwankungsbereiche liegen, die beim Methodenvergleich von Thromboplastinreagenzien normalerweise zu erwarten sind. Der für $\mathrm{He}-$ pato Quick angegebene therapeutische Bereich von 10 bis $20 \%$, der in mehreren klinischen Studien ermittelt und anerkannt wurde $(2,4,6-8,19)$, wird durch die vorliegenden Ergebnisse ebenfalls bestätigt. Allerdings zeigt sich, daß die Empfehlung differenzierter therapeutischer Bereiche mit geringerer Dosierung bei bestimmten Indikationen eine Ausweitung des therapeutischen Bereichs erforderlich macht. Die Obergrenze des therapeutischen Bereichs von Hepato Quick nach bisheriger klinischer Praxis liegt bei 20\%. Dies entspricht einer INR von etwa 2,5. Folgt man der Empfehlung einer Einstellung bis zu 2,0 , dann müßte die obere Grenze auf $28 \%$ angehoben werden. Der gleiche Effekt tritt beim Referenzthromboplastin auf, bei dem der bisher angegebene therapeutische Bereich von 5 bis $11 \%$ auf 5 bis $15 \%$ erweitert werden müßte. Aufgrund dieser Zusammenhänge erscheint eine Einstellung unter INR 2,5 insofern problematisch, als mit dieser niedrigen Dosierung bisher keine ausreichenden klinischen Erfahrungen vorliegen. Deshàlb sollten in Zukunft in sämtlichen Publikationen, in denen eine kritische Aussage zur oralen Antikoagulation, zu einer nach klinischen Gesichtspunkten unterschiedlich aggressiven Antikoagulation oder zu Kombinationen mit anderen Antithrombotika (z.B. ASS) gemacht werden, stets auch die INR als Maß für die Intensität der Antikoagulation angegeben werden. Es ist nur dann möglich, Effektivität, Nutzen und Nebenwirkungen der Behandlung unabhängig zu beurteilen und mit anderen Studien zu vergleichen, wenn man die klinischen Ergebnisse zur Intensität der oralen Antikoagulation in Beziehung setzen kann.

Die Ergebnisse machen darüber hinaus deutlich, daß zur Kontrolle der oralen Antikoagulantientherapie die nach Owren modifizierten Faktor II-, VII- und X-sensitiven Reagenzien die Methode der Wahl darstellen. Neben den bereits bekannten Vorteilen, wie gute Reproduzierbarkeit, verlängerte Probenstabilität und der patientenfreundlichen Möglichkeit des Einsatzes von Kapillarblut als Probenmaterial sowie der Verwendung von Ausgangsmaterial nichthumanen Ursprungs mit entsprechend geringer Infektionsgefahr besteht bei einem mittleren ISI von 0,95 praktisch direkte Vergleichbarkeit mit dem internationalen Referenzthromboplastin.-

\section{Schrifttum:}

1. A Double-blind Trial to Assess Longterm Oral Anticoagulant Therapy in Elderly Patients after Myocardial Infarction. Lancet 989-994 (1980).

2. BECK, E. A., MUSCHIETTI, F.: Definition des therapeutischen Bereichs der oralen Antikoagulation: Beurteilung eines neuen Test-Systems: Hepato Quick. Schweiz. med. Wschr. 104, 1218-1221 (1974).

3. BEESER, H.: Qualitätssicherung hämostaseologischer Untersuchungsmethoden. Med. Labor 29, 262-270 (1976).

4. DUCKERT, F. MARBET, G. A.: Die Kontrolle der oralen Antikoagulation - der therapeutische Bereich. Schweiz. med. Wschr. 107. 1308 (1977).

5. EISENWIENER, H. G., BABLOK, W., BARDORFF, W., BENDER, R., MARKOWETZ D.. PASSING, H., SPAETHE, R., SPECHT, W.: Präzisionsangaben beim Methodenvergleich. Lab.med. 2, 273 (1983).

6. FISCHER, M. FALKENSAMMER, Ch.: Hepato Quick - ein neues Thromboplastinzeitsystem - im Vergleich zum Normotest und Thrombotest. Wien. klin. Wschr. $86,577-583$ (1974).
7. HEENE, D. L.: Kontrolle der oralen Antikoagulantientherapie anhand des Hepato Quick-Tests. Med. Welt 25, 1529-1531 (1974).

8. HELLSTERN, P., KÖHLER, M., WENZEL, E.: Indikation und Durchführung de Antikoagulantientherapie. Therapiewoche 32, 2099-2106 (1982).

9. HERMANS, J.: The European Community Bureau of Reference Calibration Study. In: VAN DEN BESSELAAR, A. M. H. P., GRALNICK, H. R., LEWIS, S. M. (Hrsg.) Thromboplastin Calibration and Oral Anticoagulant Control. Martinus Nijhoff Publis hers, Boston, pp. $41-86$.

10. HULL, R., HIRSH, J., JAY, R., CARTER, C., ENGLAND, C., GENT, M. TURPIE, A. G. G. MCLOUGHLIN, D. DODD, P. THOMAS, M RASKOP G OCKELFORD, P.: Different Intensities of Oral Anticoagulant Therapy in the Treatment of ProximalVein Thrombosis. N. Engl. J. Med. 307, 1676-1681 (1982).

11. International Committee for Standardization in Haematology. International Committee on Thrombosis and Haemostasis. Thromb. Haemost. 53. 155-156 (1985).

12. KIRKWOOD, T. B. L.: Calibration of Reference Thromboplastins and Standardisa tion of the Prothrombin Time Ratio. Thromb. Haemostas. 49, 238-244 (1983).

13. KIRKWOOD, T. B. L.: General Aspects of Thromboplastin Calibration In: VAN DEN BESSELAAR, A. M. H. P., GRALNICK. H. R., LEWIS, S. M. (Hrsg.) Thromboplastin Calibration and Oral Anticoagulant Control. Martinus Nijhoff Publishers, Boston, pp. $11-23$.

14. LOELIGER. E. A., HENSEN, A., KROES, F., VAN DIJK, L. M., FEKKES, N., DE JONGE, H., HEMKER. H. C.: A Double-blind Trial of Long-term Anticoagulant Treatment after Myocardial Infarction. Acta med. scand. 182, 549-566 (1967).

15. LOELIGER, E. A.: Laboratory Control and Optimal Therapeutic Ranges in Oral Anticoagulant Control. Acta Haematologica 74, 125-131 (1985).

16. LOELIGER, E. A., POLLER, L., SAMAMA, M., THOMSON, J. M., VAN DEN BESSELAAR, A. M. H. P., VERMYLEN, J., VERSTRAETE, M.: Questions and Answers on Prothrombin Time Standardization in Oral Anticoagulant Control. Thromb. Haemostas. 54, 515-517 (1985)

17. LOELIGER, E. A.: Zur Arbeit „Das akute Abdomen beim antikoagulierten Patienten" von H. E. WAGNER et al. Schweiz. med. Wschr. 116, 1802-1809 (1986): Schweiz. med. Wschr. 117, 736 (1987).

18. MARBET, G. A., DUCKERT, F., CHRISTELLER, S., AIRENNE, H., KRAIJNC, V., MORNIROLI, I., MULLLER, L., FÄSSLER, H., MORGER, T.: Der therapeutische Bereich der oralen Antikoagulation. Schweiz. med. Wschr. 107, 1466 (1977).

19. MARTI, P. BECK, E. A.: Standardisierungsprobleme bei der Überwachung der oralen Antikoagulation durch die Thromboplastinzeit nach Quick: Versuch einer Standortbestimmung für die Schweiz. Schweiz. med. Wschr. 113, 1593-1597 (1983).

20. OEHLER, G., LASCH, H. G.: Antithrombotische Therapie. Inn. Med. 9, $401-409$ (1982).

21. OWREN, P. A.: The Control of Dicumarol Therapy and the Quantitative Determination of Prothrombin and Proconvertin. Scand. J. Clin. Lab. Invest. 3, $201-208$ (1951). 22. OWREN, P. A.: Thrombotest, A new Method for controlling anticoagulant therapy. Lancet II, 754-758 (1959).

23. OWREN, P. A.: The interrelationship between Normotest and Thrombotest. Farmakoterapi 25, 1-13 (1969).

24. PAAR, D.: Qualitätssicherung im Gerinnungslabor. unter besonderer Berücksichtigung der konventionellen Methoden zur Kontrolle der oralen Antikoagulantientherapie. Med. Lab. 34, 83-87 (1981).

25. PASSING, H., BABLOK, W.: A new biometrical procedure for testing the equality of measurements from two different analytical methods. J. Clin. Chem. Clin. Biochem. 21, 709-720 (1983).

26. SPANUTH, E.. BREYER, J., BABLOK, W.: Chargen- und gerätespezifische Kalibrierung der Thromboplastinzeit-Bestimmung: Ein Beitrag zur Qualitätssicherung im Labor. Lab.med. 8, 147-152 (1984).

27. SPANUTH E BREY J BABLOK, W. Erfahrungen mit einem System zur chargen- und gerätespezifischen Kalibrierung der Thromboplastinzeit (TPZ). In:WENZEL, E. et al. (Hrsg.) Rationelle Therapie und Diagnose von hämorrhagischen und thrombophilen Diathesen. F. K. Schattauer Verlag. Stuttgart, New York (S.244-248) (1986).

28. SPANUTH, E., BREYER, J.: Ergebnisse zur statistischen Qualitätskontrolle gerinnungsanalytischer Routinemethoden im Rahmen des Qualitäts-Control-Service (Q.C.S.)-Gerinnung. Lab.med. 11, 45-52 (1987).

29. VAN DEN BESSELAAR, A. M. H. P.: Standardization of the ProthrombinTime in Oral Anticoagulant Control. Haemostasis 15, $271-277$ (1985).

30. VAN DEN BESSELAAR, A. M. H. P.: Kontrolle der oralen Antikoagulantientherapie: Standardisierung der Thromboplastinzeit. Diagnose \& Labor 37, 7-14 (1987). 31. WHO Expert Committee on Biological Standardization, 33rd Report. WHO Technical Report Series 687, $81-105$ (1983).

Anschrift für die Verfasser:

Dr. E. Spanuth

Boehringer Mannheim GmbH

Abt. Blutgerinnung

Sandhofer Straße 116

6800 Mannheim 31 